\title{
A reevaluation of the effect of velocity on induced motion
}

\author{
ROBERT B. POST and DANIELLE CHI \\ University of California, Davis, California \\ THOMAS HECKMANN \\ York University, Toronto, Ontario, Canada \\ and \\ MARC CHADERJIAN \\ University of California, Davis, California
}

\begin{abstract}
Induced motion (IM) was measured as a function of the temporal frequency of inducer oscillation. IM magnitude decreased as frequency increased above $.5 \mathrm{~Hz}$. Increasing the amplitude of inducer motion, and thereby its velocity, did not influence the temporal frequency dependence of IM. This suggests that it is the duration of inducer motion, rather than its velocity, that is the critical stimulus feature in studies that report decreased IM with higher frequencies of inducer oscillation. In a separate experiment, the optokinetic nystagmus elicited by the inducing stimulus in the absence of a fixation target displayed frequency-response characteristics similar to those of IM. This finding supports the hypothesis that IM magnitude is proportional to the voluntary effort required to suppress reflexive eye movements while maintaining stable fixation.
\end{abstract}

Induced motion (IM) is the illusory motion of visual stimuli that occurs in a direction opposite the real motion of other stimuli. One stimulus parameter that is effective in influencing IM magnitude is the velocity of the inducing stimulus. In particular, Wallach and Becklen (1983) and Becklen and Wallach (1985) reported that IM magnitude decreases with increasing velocity of the inducing stimulus.

In contrast, Gogel (1979) reported little influence of velocity on IM, and Post (1986) and Post and Heckmann (1986) reported strong IM with inducer velocities far greater than those in the previously cited studies. These apparent discrepancies possibly owe to differences in the motion characteristics of inducing stimuli. In the studies of Wallach and Becklen (1983) and Becklen and Wallach (1985), the inducing stimulus oscillated. Given a constant amplitude of oscillation, the duration of motion in one direction decreases as the velocity of the stimulus increases. The inducer used by Post (1986) and Post and Heckmann (1986), however, was a set of horizontally moving vertical stripes that moved continuously and unidirectionally, such that changes in inducer velocity did not alter the duration of stimulus motion. Because IM increases with increasing duration of inducer motion

This work was supported in part by Grant D-1832 (to Robert B. Post) from the University of California. We are grateful to Rosemarie Becerra, Jennifer Buckner, and Leanna Taylor for assistance in data collection. We also thank Dwight Howard for equipment design. Address correspondence to Robert B. Post, Department of Psychology, University of California, Davis, CA 95616.
(Heckmann \& Post, 1988; Post \& Chaderjian, 1988; Post \& Heckmann, 1986), the earlier reports of decreased IM with increased velocity of an oscillating inducer may owe to the reduction of inducer duration rather than to a change in velocity. This analysis is also consistent with Gogel's (1979) lack of a velocity effect. Although Gogel used an oscillatory inducing stimulus in his study, the highest frequency investigated was below most of the frequencies of the other studies in which oscillatory inducers were used and in which IM was found to decrease with increasing frequency.

The present experiments investigated whether the decrease in IM magnitude with higher frequency stimulus oscillation results from the decreased duration of inducer motion in one direction. IM was measured with two amplitudes of inducer motion at several temporal frequencies in order to separate the factors of velocity and duration. We reasoned that if the increase of inducing stimulus velocity is the feature that decreases IM at high frequencies, then the frequency at which IM decreases should decrease when the amplitude of inducer motion (and hence velocity) is increased. Alternatively, if briefer stimulus duration at higher temporal frequencies is the feature that causes IM to decrease, then changes in the amplitude of inducer motion should not alter the frequency at which IM magnitude begins to decline. Experiments 1 and 2 used different techniques of measuring IM to investigate this hypothesis.

In Experiment 3, optokinetic nystagmus (OKN) elicited by the inducing stimulus of Experiments 1 and 2 was measured. This was done to test the hypothesis that IM 
results in part from the use of the smooth ocular pursuit system to maintain fixation by opposing OKN (Post \& Leibowitz, 1985; Post, Shupert, \& Leibowitz, 1984). Specifically, we propose that stable fixation of a stationary target in the presence of moving contours is achieved by activation of ocular pursuit in the direction opposite that of the reflexive activation elicited by the moving contours. Whereas activity in the reflexive system does not give rise to a sensation of object motion, the pursuit effort opposed to reflexive activation is registered perceptually as motion of the stationary fixation target, as if the eye were pursuing a moving target. This theory predicts that any change in stimulation that decreases the effectiveness of a moving stimulus for eliciting OKN should also reduce the amount of IM it would produce, since the pursuit effort required to oppose $\mathrm{OKN}$ and maintain fixation will also decrease. Accordingly, we predicted that any decrease in IM at higher inducer frequencies in Experiments 1 and 2 should be paralleled by a decrease in OKN at the same frequencies in Experiment 3.

\section{EXPERIMENT 1}

\section{Method}

Four individuals, aged 20 to 25 years and having at least 20/20 visual acuity (OU), served as subjects. All were naive with regard to the experimental hypotheses.

All observations were binocular. The subject was seated, facing the center of a vertically aligned hemicylinder that served as a projection surface for the IM display. A chinrest maintained head position stationary at the center of curvature of the projection screen (radius and viewing distance $=50 \mathrm{~cm}$ )

The technique for measurement of IM was based on several reports that IM and real motion summate to produce apparent motion on a path that represents a vector sum of the real and illusory motion components (see, e.g., Wallach, Bacon, \& Schulman, 1978). The apparent slope of the motion path may be used to derive the magnitude of the illusory motion component. In the present study, the inducing stimulus consisted of a horizontally moving luminous rectangle, and the fixation target (which had horizontal motion induced in it) was a vertically moving spot. The rectangle extended $14 \mathrm{~cm}$ horizontally and $7.5 \mathrm{~cm}$ vertically, with horizontal and vertical edges .5 and $.4 \mathrm{~cm}$ thick, respectively. At the viewing distance of $50 \mathrm{~cm}$, the outer edges of the inducing stimulus subtended $16^{\circ} \times 8.6^{\circ}$. $^{1}$ The image of the rectangle was projected and reflected to the hemicylindrical surface by a mirror mounted on a mirror galvanometer. The mirror galvanometer was under control of a frequency generator and amplifier circuit so that inducer frequency and amplitude were adjustable. The vertically moving fixation stimulus was formed by reflecting a laser off another mirror galvanometer. The same frequency generator circuit was connected to this galvanometer. Therefore, upward and downward motions of the spot were paired with leftward and rightward motions of the inducer, respectively.

The subjects were instructed to visually track the motion of the fixation target while attending to the apparent slope of its motion path. They then turned and rotated a knob that had a rod protruding from it so that the slope of the rod matched the apparent slope of the motion path. The rod was viewed against a featureless background, with the exception of a horizontal and a vertical stripe, so that the visible orientation reference detail matched that that was available while viewing the IM stimulus. The slope of the rod was recorded after each setting was made. Ten measures were obtained from each observer for each of six frequencies of motion (.125, $.25, .5,1.0,2.0$, and $4.0 \mathrm{~Hz}$ ) at two different amplitudes of inducer motion ( $1.7^{\circ}$ and $6.8^{\circ}$ peak-to-peak).

\section{Results and Discussion}

The horizontal component of the angular deviation of each rod setting from vertical was derived as a measure of IM. These values are presented for each subject for all frequencies and both amplitudes of inducer motion in Figure 1. It is apparent in the figure that for each subject IM is little influenced by temporal frequency at lower frequencies, and declines sharply at higher frequencies. The parallel functions obtained for the two amplitudes of inducer motion with a logarithmic ordinate indicate that frequency has the same proportional influence on IM magnitude independent of inducer motion amplitude. This finding is inconsistent with the hypothesis that IM declines at higher frequencies because of the greater velocity. If this were the case, parallel functions would not have been obtained for two different inducer amplitudes. Rather, increasing amplitude by a factor of four should have shifted by a similar factor the high-amplitude results toward lower temporal frequencies.

An analysis of variance was conducted for the results presented in Figure 1. Significant main effects were obtained for the factors of amplitude $[F(1,36)=180.99$, $p<.001]$ and frequency $[F(5,36)=28.33, p<.001]$, as well as their interaction $[F(5,36)=12.02, p<.001]$. Post hoc analyses conducted within the results obtained for each amplitude revealed that IM magnitude was similar between .125 and $.5 \mathrm{~Hz}$, and then exhibited a significant decrease with each succeeding increase in frequency [individual $F(10,180)$ values for comparisons revealing significant differences ranged from 2.28 to 5.55 ; probability values from .015 to $<.001]$. These tests therefore

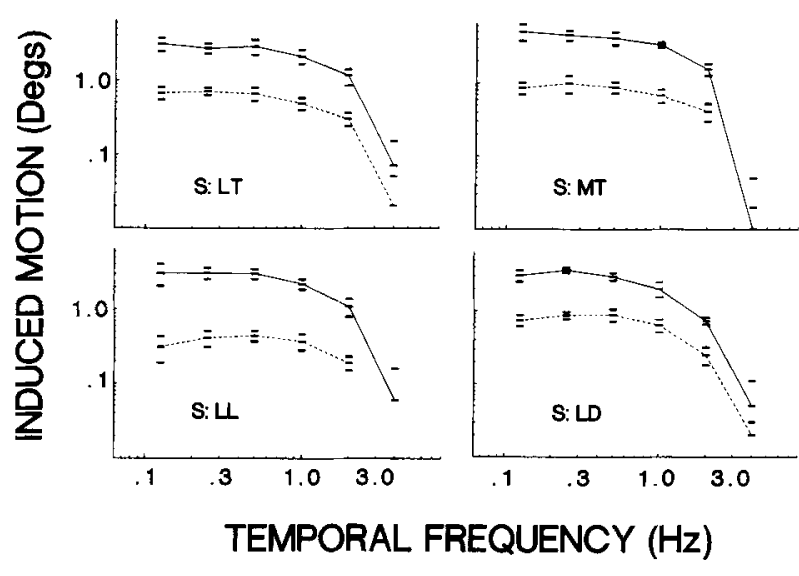

Figure 1. Induced motion (in degrees of arc) for 4 subjects with both amplitudes of inducer motion at different temporal frequencies. Plots represent the mean $\pm 1 S D$ of 10 trials. Dashed and solid lines connect the mean results with $1.7^{\circ}$ and $6.8^{\circ}$ of inducer motion, respectively. Zero and slightly negative values obtained at $4 \mathrm{~Hz}$ are not represented. The $\log -\log$ format demonstrates similar proportional attenuation at higher frequencies for both inducer amplitudes by the resulting parallel functions. 
are consistent with the impression formed by inspection of Figure 1. Specifically, the temporal frequency dependence of IM does not differ between the two amplitudes, and therefore does not reflect a sensitivity to velocity changes at higher frequencies. ${ }^{2}$

The finding of a significant effect of frequency on IM magnitude supports the hypothesis that the decrease in IM with increasing temporal frequency results from the decreased duration in stimulation rather than the increased velocity. If increased velocity is the stimulus feature responsible for the roll-off in IM at higher frequencies, then an increase in the amplitude and therefore the velocity of inducer motion by a factor of four should have reduced by a similar factor the frequency at which the IM attenuation is observed. This prediction, however, is clearly not supported.

Figure 2 contrasts the results obtained in Experiment 1 with those that would be expected on the basis of different theoretical predictions. The means of the results obtained from all subjects are presented for both amplitudes and all frequencies. Also presented is a curve that represents the high-amplitude data that would be expected on the basis of the obtained low-amplitude data, if it is assumed that it is the decrease in stimulus duration that causes the fall-off in the low-amplitude data at higher frequencies. This curve is merely displaced upward from the low-amplitude data by a factor of four (the ratio of high to low amplitudes). The other theoretical curve represents the results that would be expected in the high-amplitude condition if it is assumed that velocity causes the fall-off in the low-amplitude data at higher frequencies. This curve is displaced upward from the low-amplitude results by a factor of four (because of the increased stimulus amplitude) and shifted to the left by a factor of four (the change in velocity with increased amplitude). It is clear that the obtained high-frequency data follow the prediction made

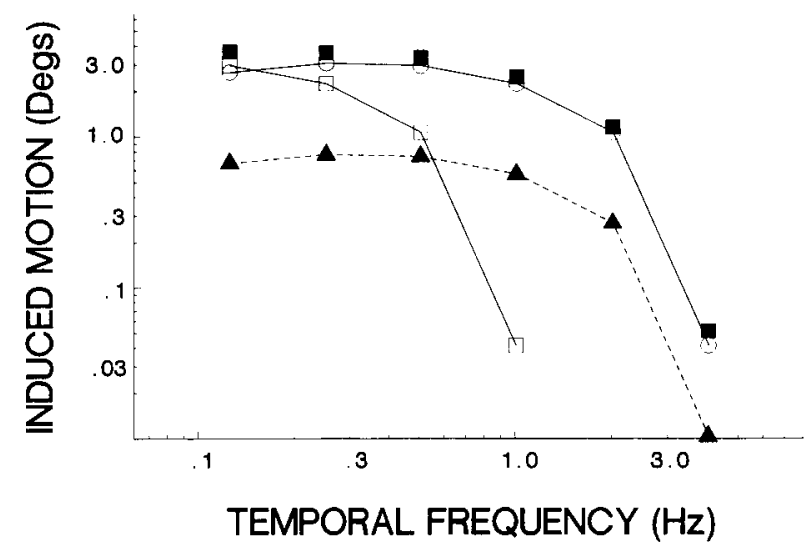

Figure 2. Means of combined subjects' data from Experiment 1 for small (triangles) and large (filled squares) amplitudes of inducer motion. Solid lines correspond to different theoretical predictions of large-amplitude results. Open squares represent predicted results if velocity is assumed to be responsible for high-frequency decay. Open circles represent predicted results if duration is assumed to be responsible for high-frequency decay. on the basis of duration, rather than on the basis of velocity.

The independence of IM of temporal frequency at low temporal frequencies is consistent with the findings of Gogel (1979). The decrease of IM at higher frequencies is consistent with the reports of Wallach and Becklen (1983) and Becklen and Wallach (1985). These authors attributed this decrease at higher frequencies to the greater velocity of high-frequency inducers. However, the present results and analysis do not support this interpretation.

\section{EXPERIMENT 2}

In Experiment 1 both the inducing stimulus and the tracked target oscillated. We had anticipated, however, that completely adequate visual tracking of the vertically moving spot was not possible at the highest frequencies used (see, e.g., Dallos \& Jones, 1963; Stark, Vossius, \& Young, 1962). To ensure that the pattern of IM decline at higher temporal frequencies obtained in Experiment 1 was not an artifact attributable to inadequate tracking, Experiment 2 was conducted as a control experiment in which subjects did not have to track a moving stimulus.

\section{Method}

The stimuli were the same as in Experiment 1, except that the fixation stimulus that had previously oscillated vertically was now stationary. In Experiment 2, lateral oscillation of the inducing stimulus therefore produced IM as apparent counterphase oscillation of the stationary fixated spot.

To quantify IM with this stimulus arrangement, the subjects fixated the stationary target and observed IM until satisfied that they could estimate the magnitude of IM in terms of apparent displacement of the stationary target. They were then given a response form that contained a rectangle the same size as the inducer. The subjects were instructed to draw a horizontal line on this form to indicate the magnitude of perceived movement of the fixated stimulus.

Six naive subjects with acuities of 20/20 or better (OU) were used. The frequencies and amplitudes of inducer motion were the same as employed in Experiment 1. Four measures were obtained from each subject at each of the temporal frequencies for both amplitudes of inducer motion.

\section{Results}

Mean lengths of the lines drawn by all subjects were computed separately for each temporal frequency and both amplitudes of motion. These values were then converted to degrees of visual angle of apparent target displacement. These values are presented separately for the two inducer amplitudes in Figure 3. IM is independent of inducer frequency up to $1 \mathrm{~Hz}$, then declines at higher frequencies. This pattern is observed with both amplitudes of inducer motion and is similar to the pattern obtained in Experiment 1 , in that the frequency at which IM magnitude begins to decrease with increasing temporal frequency does not vary with the amplitude (hence velocity) of inducer motion.

An analysis of variance of the results from Experiment 2 indicated that the main effects of inducer amplitude and frequency were significant $[F(1,60)=89.9, p<$ 


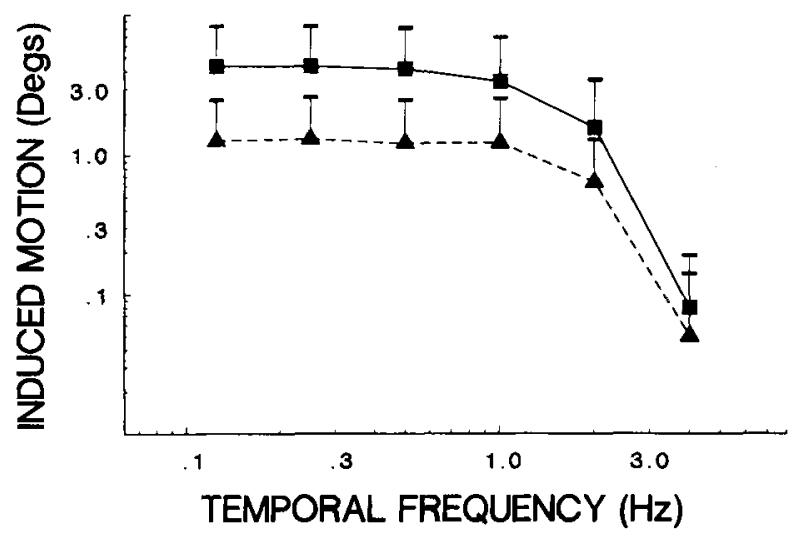

Figure 3. Induced motion (in degrees of arc) for $1.7^{\circ}$ inducer motion (triangles) and $6.8^{\circ}$ inducer motion (squares) at different temporal frequencies in Experiment 2. Plots represent the mean of responses of 6 subjects. The error bar represents $1 S D$ above the mean.

.001 , and $F(5,60)=17.69, p<.001$, respectively], as was the interaction of these factors $[F(5,60)=5.72, p<$ $.001]$. Post hoc comparisons within the results obtained for each amplitude revealed that IM magnitude was similar between .125 and $1 \mathrm{~Hz}$, and then exhibited a significant decrease with each succeeding increase in frequency [individual $F(4,120)$ values for comparisons revealing significant differences ranged from 3.02 to 7.61 ; probability values from .021 to $<.001]$. These analyses support the impression formed from inspection of Figure 3 that the relative effect of frequency on IM magnitude is independent of inducer motion amplitude, and therefore of velocity. The primary conclusion of this control experiment is therefore identical to that of Experiment 1.

It may be noted that in Experiment 2 the fall-off in IM magnitude with increasing frequency was first observed between 1 and $2 \mathrm{~Hz}$, whereas in Experiment 1 it was observed between .5 and $1 \mathrm{~Hz}$. We do not know whether this difference between studies originated from the different measurement techniques and subjects, or whether the function relating IM to temporal frequency is shifted during tracking eye movements. Whatever the cause of the finding that the location of the fall-off was slightly different in the two experiments, the results of the two experiments are in agreement with regard to the central question of this research. Specifically, it was found with widely different methodologies that changing the amplitude (hence velocity) of inducer motion does not influence the temporal frequency dependence of IM.

\section{EXPERIMENT 3}

The results of Experiments 1 and 2 indicate that the decline in IM at higher inducer frequencies is not attributable to the increased inducer velocity at these frequencies. It is more likely that the decreased duration of mo- tion of the inducer in each direction at higher frequencies is responsible for the high-frequency roll-off. This finding was expected on the basis of a theory that posits that IM results from the use of smooth ocular pursuit to suppress OKN which would otherwise be elicited by inducer motion (Post et al., 1984). In particular, high frequencies of stimulation give brief inducer motion that would not be expected to optimally activate the OKN system (Cohen, Henn, Raphan, \& Dennett, 1981; Cohen, Matsuo, \& Raphan, 1977). The amount of pursuit effort required to suppress OKN, and hence the magnitude of IM, should be correspondingly decreased (Heckmann \& Post, 1988).

Experiment 3 was conducted to investigate the hypothesis that the decrease in IM at higher inducer frequencies results from the diminished effectiveness of such inducers in activating the OKN system. Specifically, eye movement records were obtained of OKN elicited by the inducer used in Experiments 1 and 2, but without a fixation stimulus, to determine if OKN elicited by higher frequency inducers decreases in a fashion similar to the two measures of IM.

\section{Method}

Four of the subjects who served in the first two experiments participated in Experiment 3. Eye movement measures were obtained with an infrared eye monitor system (Energy Exchange, Winters, CA), with the output recorded on a Beckman strip chart recorder. Resolution of the monitoring system was $1 / 2^{\circ}$.

At the beginning of each experimental session, the eye monitor was adjusted on the subject's head to produce symmetrical responses to equivalent leftward and rightward eye movements. A series of rightward and leftward $5^{\circ}$ and $10^{\circ}$ saccades was then performed to establish a calibration record for the eye monitor system. The subjects then were instructed to look straight ahead while the inducer stimulus oscillated. The fixation stimulus from the first two experiments was not present, so no visual stimulus was available to help suppress any OKN elicited by the inducer. Thirty seconds of eye movement records were obtained from each subject at each of the temporal frequencies of inducer motion used in Experiments 1 and 2 . The amplitude of inducer motion was $6.8^{\circ}$, the same as the larger of the two amplitudes used previously. ${ }^{3}$ Stimulus position was recorded on a second channel to permit subsequent evaluation of OKN responses with regard to the direction of stimulus motion.

\section{Results and Discussion}

Measurable OKN was elicited by inducer motion frequencies below $4 \mathrm{~Hz}$. To determine the gain of OKN elicited at different frequencies, the total eye deviation during slow phase movements was computed separately for each record and divided by the total stimulus displacement during the same interval. Mean OKN gains computed across subjects for each frequency of inducer motion are presented in Figure 4.

It is apparent in the figure that the gain of OKN is fairly high and similar at frequencies below $2 \mathrm{~Hz}$. There is a drop in gain at $2 \mathrm{~Hz}$ and no measurable nystagmus at $4 \mathrm{~Hz} .{ }^{4}$ An analysis of variance was conducted for the results of the OKN measures and the effect of frequency was statistically significant $[F(5,18)=20.87, p<.001]$. 


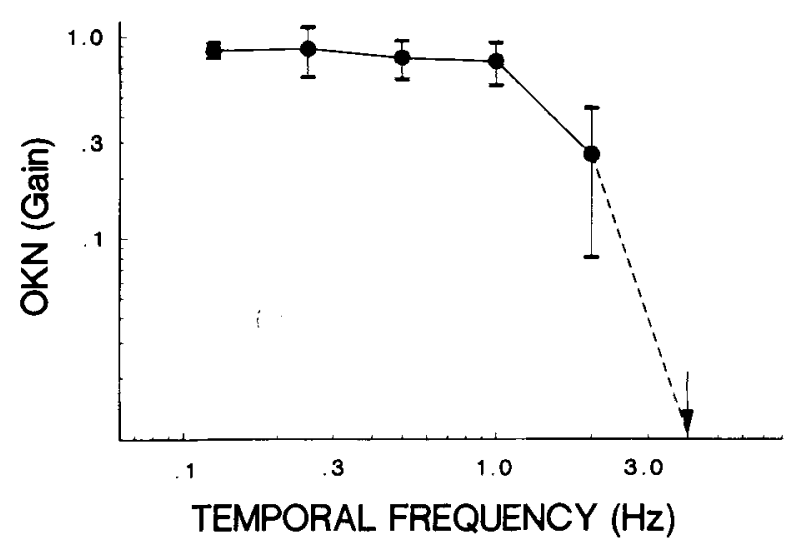

Figure 4. Gain of optokinetic nystagmus (OKN) elicited by $6.8^{\circ}$ movement of inducer stimulus in absence of a fixation stimulus at different temporal frequencies. Points represent the mean of 4 subjects, and the error hars correspond to $\pm 1 S D$. Arrow at $4 \mathrm{~Hz}$ and dashed line indicate no measurable nystagmus at $4 \mathrm{~Hz}$.

Post hoc comparisons revealed no differences in gain up to $1 \mathrm{~Hz}$, a significant decrease between 1 and $2 \mathrm{~Hz}$ $[F(1,18)=18.71, p<.001]$, and a significant decrease between 2 and $4 \mathrm{~Hz}[F(1,18)=5.32, p<.05]$. This pattern of significant differences is similar to that obtained in Experiment 1 and identical to that of Experiment 2 for the effects of frequency on IM magnitude. The similarity between the effects of frequency on OKN in Experiment 3 and the magnitude of IM in Experiments 1 and 2 was further investigated by statistical regression. IM magnitudes for large-amplitude inducer motion were highly correlated across frequency with $\mathrm{OKN}$ gain $[r(5)=.98$ and .99 for Experiments 1 and 2, respectively, both $p$ s $<.001$ ].

The highly similar dependence of IM and OKN on frequency of stimulation is consistent with the hypothesis that IM results in part from nystagmus suppression. ${ }^{5}$ According to this hypothesis, the smooth pursuit system suppresses nystagmus, which would be elicited if no fixation target were present. Activity in the smooth pursuit system in turn is perceived as movement of the fixated target (Post et al., 1984). Stimulus variables that influence the degree to which the inducing stimulus activates the OKN system therefore have a similar influence on the amount of IM observed when a fixation stimulus is present. For example, increasing the retinal eccentricity of a moving stimulus decreases both its effect on the OKN system (Cheng \& Outerbridge, 1975) and the amount of IM if a fixation target is provided (Gogel, 1974; Oppenheimer, 1935). Increasing the duration of a unidirectionally moving stimulus increases the degree to which the OKN system is activated (Cohen et al., 1981; Cohen et al., 1977) and therefore IM magnitude (Post \& Heckmann, 1986). Changes in stimulus luminance and velocity also exert similar influences on IM and optokinetic afternystagmus (Post, 1986). The present study extends the list of stimulus parameters that similarly affect reflex- ive gaze responses and IM to the temporal frequency of the inducing stimulus.

\section{REFERENCES}

Becklen, R., \& Wallach, H. (1985). How does speed change affect induced motion? Perception \& Psychophysics, 37, 231-236.

Cheng, M., \& Outerbridge, J. S. (1975). Optokinetic nystagmus during selective retinal stimulation. Experimental Brain Research, 23, 129-139.

Cohen, B., Henn, V., Raphan, T., \& Dennett, D. (1981). Velocity storage, nystagmus, and visual-vestibular interactions in humans. Annals of the New York Academy of Science, 374, 421-433.

Cohen, B., Matsuo, V., \& RAPHan, T. (1977). Quantitative analysis of the velocity characteristics of optokinetic nystagmus and optokinetic after-nystagmus. Journal of Physiology (London), 270, 321-344.

Dallos, P. J., \& Jones, R. W. (1963). Learning behavior of the eye fixation control system. IEEE Transactions on Automatic Control, 8 , 218-227.

GoGEL, W. C. (1974). Relative motion and the adjacency principle. Quarterly Journal of Experimental Psychoiogy, 26, 425-437.

GoGEL, W. C. (1977). Independent motion induction in separate portions of the visual field. Bulletin of the Psychonomic Society, 10, 408-415.

GoGEL, W. C. (1979). Induced motion as a function of the speed of the inducing object, measured by means of two methods. Perception, $8,255-262$.

HeckmanN, T., \& Post, R. B. (1988). Induced motion and optokinetic afternystagmus: Parallel response dynamics with prolonged stimulation. Vision Research, 28, 681-694.

Johansson, G. (1977). Studies in visual perception of locomotion. Perception, 6, 365-376.

Nakayama, K., \& Tyler, C. W. (1978). Relative motion induced between stationary lines. Vision Research, 18, 1663-1668.

OPPENHEIMER, E. (1935). Optische Versuche über Ruhe und Bewegung. Psychologische Forschung, 20, 1-46.

Post, R. B. (1986). Induced motion considered as a visually induced oculogyral illusion. Perception, 15, 131-138.

Post, R. B., Chaderian, M. (1988). The sum of induced and real motion is not a straight path. Perception \& Psychophysics, 43, 121-124.

Post, R. B., \& Heckmann, T. (1986). Induced motion and apparent straight-ahead during prolonged stimulation. Perception \& Psychophysics, 40, 263-270.

Post, R. B., \& Leibowitz, H. W. (1985). A revised analysis of the role of efference in motion perception. Perception, 14, 631-643.

Post, R. B., Shupert, C., \& Leibowitz, H. W. (1984). Implications of OKN suppression by smooth pursuit for induced motion. Perception \& Psychophysics, 36, 493-498.

Stark, L., Vossius, G., \& Young, L. R. (1962). Predictive control of eye tracking movements. IRE Transactions on Human Factors in Electronics, 3, 52-57.

Wallach, H., Bacon, J., \& Schulman, P. (1978). Adaptation in motion perception: Alteration of induced motion. Perception \& Psychophysics, 24, 509-514.

WALLACH, H., \& BECKLEN, R. (1983). An effect of speed on induced motion. Perception \& Psychophysics, 34, 237-242.

\section{NOTES}

1. The precise spatial characteristics of the inducing stimulus do not appear to be critical for the pattern of psychophysical results obtained. During pilot measures, a slide of a vision scientist and his daughters was used as an inducer, and IM magnitude was found to be influenced by temporal frequency in a manner similar to that reported in this paper.

2. An alternate data analysis was also performed in which results obtained with the different amplitudes were first normalized to eliminate differences in overall scale. These normalized data were then subjected to an analysis of variance, which revealed no significant interaction of 
amplitude and frequency. This analysis therefore also supports the conclusion that frequency has the same proportional effect on IM regardless of amplitude of inducer motion.

3. The smaller inducer amplitude, $1.7^{\circ}$, was not used in Experiment 3 because the resulting $\mathrm{OKN}$ would be correspondingly smaller and more difficult to measure.

4. It is possible that $\mathrm{OKN}$ was elicited with 4-Hz stimulation, but the amplitude was below the resolution of the eye monitoring system. This would be expected on the basis of the high degree of attenuation of the psychophysical measures at $4 \mathrm{~Hz}$.
5. We believe that a variety of mechanisms may contribute to IM under different viewing conditions. For example, simultaneous bidirectional IM (Gogel, 1977; Nakayama \& Tyler, 1978) argues for a contribution of relative motion, and observations such as those of Johansson (1977) suggest a role for visual capture of vection. The present results support a nystagmus suppression hypothesis.

(Manuscript received July 11,1988 ; revision accepted for publication October $12,1988$. ) 